Check for updates

Cite this: Sens. Diagn., 2022, 1, 193

Received 21st September 2021 Accepted 18th November 2021

DOI: $10.1039 / \mathrm{d} 1 \mathrm{sd00021 \textrm {g }}$

rsc.li/sensors

\title{
A novel time-resolved fluorescent lateral flow immunoassay for quantitative detection of the trauma brain injury biomarker-glial fibrillary acidic protein $\dagger$
}

\author{
Satheesh Natarajan (iD *a and Jayaraj Joseph ${ }^{\mathrm{b}}$
}

\begin{abstract}
A highly sensitive time-resolved fluorescence lateral flow immunoassay (TRF-LFIA) was developed to quantify glial fibrillary acidic protein (GFAP), a trauma brain injury (TBI) biomarker in blood, for the purpose of providing a diagnosis of mild brain injury. The assay detection limit was $10 \mathrm{pg} \mathrm{mL}^{-1}$ with a diagnostics range of $0-400 \mathrm{pg} \mathrm{mL}^{-1}$. The assay coefficients of variation (CoVs) were less than $10 \%$. Availability of the TRF-LFIA for blood testing would produce a paradigm shift in TBI patient management and clinical outcomes in trauma departments. Due to its capacity to measure low levels of blood biomarkers in human fluids, this type of assay is expected to find use in the rapid detection of various human disorders.
\end{abstract}

\section{Introduction}

Traumatic brain injury (TBI) is a silent neuro-trauma epidemic, ${ }^{1}$ caused by a mechanical effect that is applied to the head. About $27 \mathrm{~m}$ new cases of TBI were reported worldwide in $2016 .^{2}$ Annually, $\sim 1 \mathrm{~m}$ people are injured and $\sim 20000$ people die of TBI in India. ${ }^{3}$ TBI faces diagnostic and therapeutic challenges, especially in the mild injury range.

TBI can be classified as mild, moderate, or severe, based on the 15-point scale Glasgow Coma Scale (GCS) score. ${ }^{4}$ GCS evaluates eye opening, motor response, and verbal response, often a subjective approach for TBI diagnosis. ${ }^{5}$ A confirmatory report on moderate or severe TBI patients is performed with computed tomography (CT), but its high costs and requirement of being operated by trained technicians have limited its use in rural facilities and military settings. In addition, clinical CT often fails to detect mild $\mathrm{TBI}^{6}$ and cannot predict ongoing secondary brain injury. Also, magnetic resonance imaging (MRI) is more sensitive than CT, but MRI is not used in emergency cases due to its high cost and low efficacy in detecting bleeds and fractures. So the current methods of TBI assessment are tedious and expensive.

A quantitative approach involving testing a blood-based biomarker, to predict early neurological deterioration, can

\footnotetext{
${ }^{a}$ Healthcare Technology Innovation Center, Indian Institute of Technology, Madras, 600113, India.E-mail: satheesh@htic.iitm.ac.in

${ }^{b}$ Department of Electrical Engineering, Indian Institute of Technology, Madras, 600036, India

$\dagger$ Electronic supplementary information (ESI) available. See DOI: 10.1039/ d1sd00021g
}

overcome the subjective approach of GCS. Examples of blood biomarkers that are elevated in the case of TBI include glial fibrillary acidic protein (GFAP), S-100 $\beta$, neuron-specific enolase (NSE), and myelin basic protein (MBP) ${ }^{7-9}$ GFAP is a glial cell injury biomarker, and is released from glial cells upon their being damaged and then appears in the blood. So GFAP can be detected in blood samples shortly after the damage. ${ }^{10,11}$ In the case of mild and moderate cases of TBI, GFAP levels have been found to be increased eight hours after the trauma. ${ }^{12}$ In addition, the concentration of GFAP in the blood has also been suggested to predict the outcome of the injury. ${ }^{13}$ Furthermore, one test that measures GFAP (and UCH-L1) has been approved by the Food and Drug Administration for evaluating mild TBI. ${ }^{14}$

An increasing number of studies have indicated that GFAP might be a useful biomarker for differentiating between hemorrhagic and ischemic strokes. Both types of strokes can have severe consequences, but since they form using different mechanisms, they require different treatment strategies. Therefore, it is important to find tools that help in differentiating between the two strokes as early as possible. Studies have shown that GFAP levels start increasing within two hours of the onset of a hemorrhagic stroke, and peak between 6 and 12 hours after the onset of the stroke. In contrast, in the case of an ischemic stroke, the GFAP levels in the blood increase at a later time point. ${ }^{15,16}$ Thus, GFAP is a potential tool in the diagnosis and treatment of brain injury.

To realize the diagnostic potential of GFAP, we have applied the time-resolved fluorescence lateral flow immunoassay (TRF-LFIA) as an ultrasensitive method to detect GFAP in blood samples. For the TRF-LFIA, we used 
carboxylate-modified polystyrene $\mathrm{Eu}(\mathrm{III})$ chelate microparticles (CM-EUs) as a reporter. Unlike fluorescein, europium complexes show a large Stokes shift, and hence a resulting minimization of auto-fluorescence, light absorption, and light scattering by the blood matrix. Also, the fluorescence displayed by europium complexes was found in the current work to be relatively long lived, a feature allowing its measurement following excitation to be delayed and hence endowing the TRF-LFIA with a strong resistance to being interfered with by non-specific molecules in complex sample matrices. The cut-off value of GFAP in the blood of acute TBI patients was determined to be $150 \mathrm{pg} \mathrm{mL}^{-1}$ in blood for acute TBI patients. ${ }^{17}$ This TRF-LFIA assay was found to be rapid, sensitive, and highly discriminating between normal controls and head injury patients. Furthermore, this assay showed good sensitivity and stability and was determined to be extremely suitable for rapid detection in early diagnosis, and to have great potential in clinical applications.

\section{Materials and methods}

\section{Materials}

Carboxylate-modified polystyrene Eu(III) chelate microparticles (CM-EU) were purchased from Thermo Fisher Scientific. $N$-Hydroxysulfosuccinimide sodium salt (sulfoNHS), $N$-(3-dimethyl aminopropyl)- $N$-ethyl carbodiimide hydrochloride (EDC), bovine serum albumin (BSA), goat antimouse IgG, phosphate-buffered saline (PBS), phosphate buffer (PB), NaOH, sucrose, Tris-HCl, Tween-20, and Triton X-100 were procured from Sigma-Aldrich, USA. Nitrocellulose membranes (Hi-Flow 70 plus) were procured from Merck Millipore (Bedford, MA, USA). Sample pad Gx (plasma separation pad) products were obtained from the Pall Corporation (USA), and conjugation pad (GFDx) and absorbent pad (CF6) products from Whatman (GE Healthcare, UK). The anti-GFAP capturing antibody, the antiGFAP detecting antibody, and recombinant protein GFAP were obtained from HyTest Ltd, Finland.

\section{Buffer solution}

Sample pad treatment buffer (0.1 M PBS, 0.5\% BSA, 0.05\% Triton X-100), conjugate pad treatment buffer (0.5\% BSA, and $0.05 \%$ Triton $\mathrm{X}-100$ in $0.1 \mathrm{M}$ phosphate buffer, $\mathrm{pH} 7.4$ ), activating buffer (0.05 M MES, pH 5.1), binding buffer $(0.01$ $\mathrm{M}$ phosphate buffer, $\mathrm{pH}$ 7.0), washing buffer (0.2\% Tween-20, $0.9 \% \mathrm{NaCl}$ in $0.25 \mathrm{M}$ Tris- $\mathrm{HCl}, \mathrm{pH} 7.4)$, storage buffer $(1 \%$ BSA, $5 \%$ trehalose, $20 \%$ sucrose in $0.25 \mathrm{M}$ Tris- $\mathrm{HCl}$, pH 9.0), assay chase buffer $(1.5 \%$ BSA, $0.3 \%$ Triton $\mathrm{X}-100$, in $0.1 \mathrm{M}$ $\mathrm{PB}, \mathrm{pH} 7.4$ ), and all other solutions used in this study were freshly prepared before use.

The key methods used in the development of the GFAP LFIA kit are explained in the following sections.

\section{Functionalizing CM-EUs with antibodies}

Briefly, $12 \mathrm{mM}$ of EDC and $9 \mathrm{mM}$ of sulfo-NHS were added to $2 \mathrm{mg}$ of CM-EU nanoparticles suspended in the activation buffer $1 \mathrm{~mL}$. The resulting mixture was incubated for 30 minutes with gentle stirring at room temperature. To remove unreacted components, the CM-EUs were washed three times by subjecting the mixture to centrifugation at $14000 \mathrm{~g}$. The resulting pellet was ultrasonically suspended in a binding buffer. A mass of $50 \mu \mathrm{g}$ of anti-GFAP was added to the activated CM-EUs. After 4 hours of incubation, the functionalized CM-EUs were washed thrice before being combined with blocking buffer (2\% BSA in $25 \mathrm{mM}$ phosphate buffer, $\mathrm{pH}$ 7.4). The antibody-conjugated CM-EUs were washed three times with washing buffer and were suspended in storage buffer.

\section{Fabrication of GFAP lateral flow immunoassay membranes}

The LFIA membrane strip consisted of a sample application, a conjugate pad, an NC membrane, and an absorbent pad. To the conjugate pad was added the conjugation mixture diluted from the original stock $\left(10 \mu \mathrm{g} \mathrm{mL} \mathrm{m}^{-1}\right)$ to different concentrations $\left(0.5,1.5,2 \mathrm{ng} \mathrm{mL^{-1 }}\right)$ and dried at $37{ }^{\circ} \mathrm{C}$ for 1 hour. The capture antibody (a) $0.5 \mathrm{mg} \mathrm{ml}^{-1}$ and the goat antimouse IgG @ $0.5 \mathrm{mg} \mathrm{mL}^{-1}$ were dispensed onto the $\mathrm{NC}$ membrane for the test and control lines and dried at $37{ }^{\circ} \mathrm{C}$ for 1 hour. Finally, the membranes were cut each to a width of $3.1 \mathrm{~mm}$ and stored at $4{ }^{\circ} \mathrm{C}$.

\section{Lateral flow assay procedure}

In this proposed study, GFAP recombinant proteins were prepared in a dilution of GFAP $0,10,50,100,200,300$ and $400 \mathrm{pg} \mathrm{mL} \mathrm{m}^{-1}$ in $0.01 \mathrm{M} \mathrm{PB}, \mathrm{pH} 7.4+1 \%$ casein $+0.25 \%$ Tween-20 + $1 \mathrm{mM}$ EDTA. For the assay development, a volume of $85 \mu \mathrm{l}$ of the sample buffer along with the protein was dropped onto the sample pad packed in the cartridge. After 15 minutes, the data were acquired by using an IQuantTRF Immunoanalyser. ${ }^{18}$ The signal generated in the NC membrane was scanned by the instrument to quantify the biomarker. The fluorescence signals at the test $(T)$ line and control $(C)$ line were used to calculate the $T / C$ volume ratio. The entire assay was done in triplicate, and the corresponding mean volume ratio $\left(V_{\mathrm{R}}\right)$ value was plotted against the GFAP concentration to obtain the calibration curve.

\section{Determining the limit of blank and limit of detection}

The limit of detection (LOD) was estimated according to the Clinical \& Laboratory Standards Institute (CLSI) guidelines. To calculate the LOD, six samples with the lowest concentration of GFAP were measured in triplicate for five consecutive days. The values of LOB, i.e., for the negative samples, and LOD were obtained by using the expression $1.645 \times \mathrm{SD}$ and the equation $\mathrm{LOD}=\mathrm{LOB}+1.645$ [SD lower level of the sample], respectively.

\section{Statistical analysis}

All quantitative data describing the sensitivity of the assay were analyzed using Graph Pad Prism 6.0 (Graph Pad 
software, La Jolla, CA, USA). Each parameter was calculated using a 95\% confidence interval (CI) and the coefficient of variation $(\mathrm{CV})$ of Cutt-off Index (COI) values was also analyzed for the assay data. The values of LOB, i.e., for the negative samples, and LOD were obtained by using the expression $1.645 \times \mathrm{SD}$ and the equation $\mathrm{LOD}=\mathrm{LOB}+1.645[\mathrm{SD}$ lower level of the sample], respectively. ${ }^{19}$

\section{Results and discussion}

\section{The principle of the TRF-lateral flow immunoassay}

The TRF-LFIA test strip developed in this study was a sandwich module. As illustrated in Fig. 1, any GFAP antigen present in the sample migrated to the test membrane through capillary action and then bound with CM-EUs-antiGFAP detection antibody $\left(D_{\mathrm{AB}}\right)$ in the conjugation pad to form CM-EUs- $D_{\mathrm{AB}}$-GFAP-Ag. The CM-EUs- $D_{\mathrm{AB}}-\mathrm{Ag}$ then continued to flow along the membrane, to react in the test line where CM-EUs- $D_{\mathrm{AB}}-\mathrm{Ag}$ selectively reacted with $C_{\mathrm{AB}}$-GFAP to form a CM-EUs- $D_{\mathrm{AB}}$-GFAP-Ag- $C_{\mathrm{AB}}$-GFAP complex. Excess CM-EUs- $C_{\mathrm{AB}}$-GFAP flowed further and reacted with the control line. After the completion of the reaction, the test strip was analyzed using an IQuant-TRF reader by measuring the volumes of the test line $\left(V_{\mathrm{T}}\right)$ and the control line $\left(V_{\mathrm{C}}\right)$ to calculate the $V_{\mathrm{R}}$ (volume ratio). The fluorescence intensity in the test line was directly correlated with the levels of GFAP in the sample. But the fluorescence intensity at the control line remained nearly constant for the entire test.

\section{Optimization of the experimental parameters}

LFIA parameters for detecting GFAP were first optimized in pure buffers in the absence of blood. Sample solutions containing different concentrations of GFAP were prepared in the running buffer. The parameters optimized included (a) running buffers, (b) casein percent, (c) Tween-20 percent and (d) amount of EDTA. Fig. S2† (a) shows the amount of detection antibody $\left(D_{\mathrm{AB}}\right)$ and (b) amount of capture antibody
$\left(C_{\mathrm{AB}}\right)$ on the test line. Fig. S5† (a) shows the volume of blood in the buffer and Fig. S5† (b) shows the incubation time. Respective data and figures (see ESI $\dagger$ and Fig. S1, S2 and S5) are given in the ESI. $\dagger$ The best results were obtained when (a) using a running buffer of phosphate buffer $(0.01$ M PB, pH 7.4; 1\% casein; 0.25\% Tween; 1 mM EDTA), (b) using CM-EUs- $D_{\mathrm{AB}}$ with $0.5 \mathrm{ng}$ of conjugates, and (c) dispensing $1.0 \mathrm{mg} \mathrm{m} \mathrm{m}^{-1}$ of $C_{\mathrm{AB}}$ onto the test line. To construct the calibration curve, each sample was examined three times and the average intensity of each concentration was used to draw the calibration curve (Fig. S4a $\dagger$ ). A standard curve was obtained based on the measurement of six serial standards $(0,10,50,100,200,300$, and $400 \mathrm{pg}$ $\left.\mathrm{mL}^{-1}\right)$. The standard curve was produced by plotting the $V_{\mathrm{R}}$ (y) against the GFAP concentration $(x)$. Under these optimized conditions, we obtained a reasonable calibration curve for the proposed assay (Fig. S4a $\dagger$ ). The expression for the regression curve was $5 \times 10^{-6} x^{2}+0.0033 x+0.0295, n=$ $10,(r=0.9892)$. The analytical sensitivity of the proposed assay, defined as the mean plus two SD $(n=10)$ of the zero standards, was $5 \mathrm{pg} \mathrm{mL}^{-1}$. These results showed the excellent performance of the developed CM-EU test strips. Fig. $\mathrm{S} 4 \mathrm{~b} \dagger$ presents typical photographic images (left) and corresponding $V_{\mathrm{R}}$ (right) of the LFIA with increasing GFAP concentrations ( $0 \mathrm{pg}$ to $400 \mathrm{pg} \mathrm{mL}^{-1}$ ) under the optimized experimental conditions. No band was observed on the LFIA test zone in the absence of GFAP (control, $0 \mathrm{pg} \mathrm{mL} \mathrm{m}^{-1}$ ), indicating negligible nonspecific adsorption. The test band intensity increased with increasing GFAP concentration. The test band was still seen at GFAP levels as low as $7.5 \mathrm{pg}$ $\mathrm{mL}^{-1}$.

\section{Precision}

The reproducibility of the assay was examined by testing six

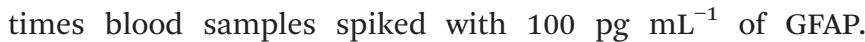
Reproducible signals were obtained with a relative standard deviation (RSD) of $5.8 \%$.

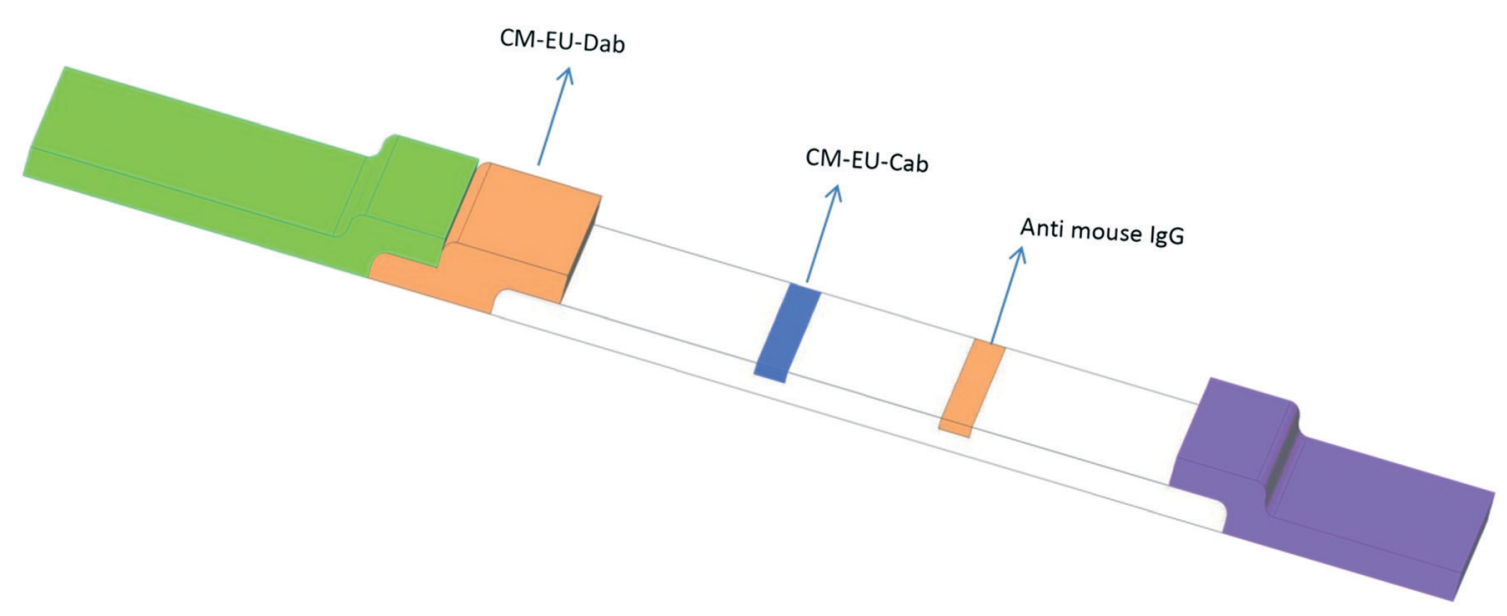

Fig. 1 Schematic presentation of the structure of the lateral flow immunoassay (LFIA) strip for the determination of GFAP levels. 


\section{Analysis of GFAP in a spiked blood sample}

Blood samples containing different concentrations of GFAP were prepared by spiking GFAP standards. The volume of CM-EUs- $D_{\mathrm{AB}}$ conjugates, the volume of blood used per assay, and the incubation time of CM-EUs- $D_{\mathrm{AB}}$ in blood were optimized to obtain the best results (Fig. S5b†). The best results were obtained when a volume of fifteen microliters of human blood was spiked with a desired concentration of GFAP and then mixed with $85 \mu \mathrm{l}$ of running buffer $(0.01 \mathrm{M}$ $\mathrm{PB}, \mathrm{pH} 7.4+1 \%$ casein $+0.25 \%$ Tween-20 +1 mM EDTA). The mixture was incubated for 10 minutes under general shaking and then applied to the LFIA sample pad. As shown in Fig. S5a, $\dagger$ a test band was observed even for a GFAP concentration of $7.5 \mathrm{pg} \mathrm{mL} \mathrm{m}^{-1}$. No obvious band was observed in blood without spiking the GFAP. The intensities of the LFIA test bands increased as the concentration of spiked GFAP in the blood was increased from 0 to $400 \mathrm{pg} \mathrm{mL}^{-1}$ (Fig. $\mathrm{S} 5 \mathrm{~b} \dagger)$. The limit of detection for GFAP was $7.5 \mathrm{pg} \mathrm{mL} \mathrm{mL}^{-1}$, which should be good enough for diagnosing TBI due to its GFAP cut-off value being about $5 \mathrm{pg} \mathrm{mL}^{-1}$ in the blood of healthy people. The reproducibility of the assay was also studied: blood samples spiked with $100 \mathrm{pg} \mathrm{mL}{ }^{-1}$ of GFAP were tested six times and gave reproducible signals with a relative standard deviation (RSD) of $<10 \%$ (Fig. S3†).

\section{Discussion}

Compared to GFAP might be a more specific and promising marker for producing a diagnosis as well as monitoring the efficacy of neuroprotective treatment in patients with CNS and concomitant extra cerebral disorders. The value of the GFAP in the pg $\mathrm{mL}^{-1}$ increased from the day of admission to the fourth day after stroke and the concentration of GFAP was significantly correlated at days 2,3 , and 4 . GFAP is a monomeric intermediate filament protein expressed almost exclusively by astrocytes. Therefore, GFAP might be a promising new and relatively specific marker for astrocytic damage in blood. The newly developed CM-EUs-sandwich immunofluorescent assay test can be completed within 25 minutes and can provide quantitative results on site when used with a portable reader device.

The sandwich-type CM-EUs lateral flow assay presented here is the first such assay based on commercially available antibodies. The sensitivity of the assay was shown to be 10 $\mathrm{pg} \mathrm{mL}^{-1}$, with the standard curve ranging from 0 to $400 \mathrm{pg}$ $\mathrm{mL}^{-1}$.

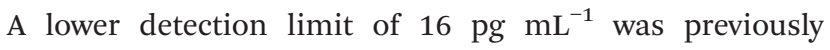
achieved using a sandwich ELISA based on anti-GFAP antibodies, ${ }^{20,21}$ and a detection limit of $<10 \mathrm{pg} \mathrm{mL}^{-1}$ was previously achieved with a dissociation-enhanced lanthanide fluorescence immunoassay (DELFIA) system. ${ }^{22}$ But that ELISA-based assay requires more time than the currently developed system and is laborious. Missler et al. reported a very sensitive assay but also with relatively weak signals for positive blood samples from head injury patients. ${ }^{23}$ They found increased GFAP levels in 12 out of 25 blood samples from head injury patients, with expression levels approximately 10-fold lower than those for the biomarker S100B. In addition, GFAP could be detected $24 \mathrm{~h}$ after injury in only 1 out of 25 blood samples while others showed comparable blood GFAP and S100B levels and found a GFAP peak at $24 \mathrm{~h}$ after head injury. ${ }^{24}$

The evaluation of the CM-EUs-LFIA-GFAP assay showed that GFAP concentrations in patients with acute stroke are highly and significantly associated with the severity of the neurological disorder and functional status at discharge from the hospital. ${ }^{25}$ Thus, CM-EUs-LFIA-GFAP values were determined to be correlated with the development of the disease and with clinical outcomes. The comparative analysis of serial GFAP blood concentrations with stroke patients demonstrated the highly reliable clinical performance of the CM-EUs-LFIA-GFAP assay. Taken together, our data demonstrated that the CM-EUs-LFIAGFAP assay is a powerful tool to detect GFAP in blood, even at low levels. Furthermore, the CM-EUs-LFIA platform makes it possible to determine GFAP levels at a point of need, where the availability of a laboratory with costly equipment is limited, and also to determine these levels even when only a small volume of sample from the patient is available.

\section{Conclusions}

A rapid, low-cost, and sensitive approach involving the use of a TRF-LFIA strip was developed to detect GFAP in blood. The CM-EUs consisted of the carboxyl group with a large Stokes shift, which produces less background noise. The demonstrated low $7.5 \mathrm{pg} \mathrm{mL} \mathrm{mL}^{-1}$ limit of detection for GFAP in the clinical blood samples makes this LFA a truly earlydiagnosis kit for stroke. The precision of the assay according to the CV from the intra-assay and inter-assay were $<10 \%$. To the best of our knowledge, this work was the first time that a point-of-care lateral flow assay for the detection of GFAP in blood samples was developed. Further work will aim to improve the detection limit of the assay and to extend the assay to being able to analyze, on a large scale, blood samples from healthy control and trauma brain injury patients at different stages.

\section{Conflicts of interest}

There are no conflicts to declare.

\section{References}

1 J. Zhang, R. Wang, H. Wang, Y. Li, H. Zhang, L. Dong and $\mathrm{H}$. Zhang, Activation of brain regions using task-state FMRI in patients with mild traumatic brain injury: a meta-analysis, Int. J. Clin. Exp. Pathol., 2020, 13(12), 2918-2926.

2 S. Y. $\mathrm{Ng}$ and A. Y. W. Lee, Traumatic Brain Injuries: Pathophysiology and Potential Therapeutic Targets, Front. Cell. Neurosci., 2019, 13, 528. 
3 G. Gururaj, Epidemiology of Traumatic Brain Injuries: Indian Scenario, Neurol. Res., 2002, 24(1), 24-28, n.d.

4 R. Sharma and D. T. Laskowitz, Biomarkers in Traumatic Brain Injury, Curr. Neurol. Neurosci. Rep., 2012, 12(5), 560-569, n.d.

5 H. S. Levin and R. R. Diaz-Arrastia, Diagnosis, Prognosis, and Clinical Management of Mild Traumatic Brain Injury, Lancet Neurol., 2015, 14(5), 506-517, n.d.

6 K. Bettermann and J. E. Slocomb, Clinical Relevance of Biomarkers for Traumatic Brain Injury, Biomarkers for Traumatic Brain Injury, 2012, vol. 1, p. 18, n.d.

7 D. V. Agoston, A. Shutes-David and E. R. Peskind, Biofluid Biomarkers of Traumatic Brain Injury, Brain Inj., 2017, 31(9), 1195-1203, n.d.

8 E. Gordillo-Escobar, et al. Usefulness of Biomarkers in the Prognosis of Severe Head Injuries, Med. Intensiva, 2016, 40(2), 105-112, n.d.

9 K. K. Wang, et al. An Update on Diagnostic and Prognostic Biomarkers for Traumatic Brain Injury, Expert Rev. Mol. Diagn., 2018, 18(2), 165-180, n.d.

10 C. Foerch, et al. Diagnostic Accuracy of Plasma Glial Fibrillary Acidic Protein for Differentiating Intracerebral Hemorrhage and Cerebral Ischemia in Patients with Symptoms of Acute Stroke, Clin. Chem., 2012, 58, 237-245, n.d.

11 L. Papa, et al. Time Course and Diagnostic Accuracy of Glial and Neuronal Blood Biomarkers GFAP and UCH-L1 in a Large Cohort of Trauma Patients with and without Mild Traumatic Brain Injury, JAMA Neurol., 2016, 73, 551, n.d.

12 P. E. Vos, et al. GFAP and S100B Are Biomarkers of Traumatic Brain Injury: An Observational Cohort Study, Neurology, 2010, 75, 1786-1793, n.d.

13 P. E. Vos, et al. GFAP and S100B Are Biomarkers of Traumatic Brain Injury: An Observational Cohort Study, Neurology, 2010, 75, 1786-1793.

14 U.S. Food \& Drug Administration, FDA Authorizes Marketing of First Blood Test to Aid in the Evaluation of Concussion in Adults, FDA, 2018, at <http://www.Fda.Gov/News-Events/ Press-Announcements/Fda-Authorizes-Marketing-FirstbloodTest-Aid-Evaluation-Concussion-Adults $>$, n.d.
15 C. Foerch, et al. Diagnostic Accuracy of Plasma Glial Fibrillary Acidic Protein for Differentiating Intracerebral Hemorrhage and Cerebral Ischemia in Patients with Symptoms of Acute Stroke, Clin. Chem., 2012, 58, 237-245.

16 U.S. Food \& Drug Administration, FDA Authorizes Marketing of First Blood Test to Aid in the Evaluation of Concussion in Adults, FDA, 2018, at <http:/www.Fda.Gov/News-Events/ Press-Announcements/Fda-Authorizes-Marketing-FirstbloodTest-Aid-Evaluation-Concussion-Adults $>$.

17 L. Papa, et al. Time Course and Diagnostic Accuracy of Glial and Neuronal Blood Biomarkers GFAP and UCH-L1 in a Large Cohort of Trauma Patients with and without Mild Traumatic Brain Injury, JAMA Neurol., 2016, 73, 551.

18 S. Karthik, et al. A Motion Free Image Based TRF Reader for Quantitative Immunoassay, 2019 IEEE Healthcare Innovations and Point of Care Technologies, (HI-POCT), IEEE, 2019, n.d.

19 K. Glynou, et al. Oligonucleotide-Functionalized Gold Nanoparticles as Probes in a Dry-Reagent Strip Biosensor for DNA Analysis by Hybridization, Anal. Chem., 2003, 75(16), 4155-4160, n.d.

20 L. E. Rosengren, et al. A Sensitive ELISA for Glial Fibrillary Acidic Protein: Application in CSF of Children, J. Neurosci. Methods, 1992, 44(2-3), 113-119, n.d.

21 L. E. Rosengren, C. Wikkelsø and L. Hagberg, A Sensitive ELISA for Glial Fibrillary Acidic Protein: Application in CSF of Adults, J. Neurosci. Methods, 1994, 51(2), 197-204, n.d.

22 U. Missler, et al. Measurement of Glial Fibrillary Acidic Protein in Human Blood: Analytical Method and Preliminary Clinical Results, Clin. Chem., 1999, 45(1), 138-141, n.d.

23 U. Missler, et al. Measurement of Glial Fibrillary Acidic Protein in Human Blood: Analytical Method and Preliminary Clinical Results, Clin. Chem., 1999, 45(1), 138-141.

24 M. Herrmann, et al. Release of Glial Tissue-Specific Proteins after Acute Stroke: A Comparative Analysis of Blood Concentrations of Protein S-100B and Glial Fibrillary Acidic Protein, Stroke, 2000, 31, 2670-2677, n.d.

25 L. Papa, Time Course and Diagnostic Accuracy of Glial and Neuronal Blood Biomarkers GFAP and UCH-L1 in a Large Cohort of Trauma Patients with and without Mild Traumatic Brain Injury, JAMA Neurol., 2016, 73, 551, n.d. 